\title{
EFEITOS DA RADIOFREQUÊNCIA NO TRATAMENTO DA FLACIDEZ DOS GRANDES LÁBIOS DO PUDENDO DA MULHER MENOPAUSADA
}

\section{EFFECTS OF RADIOFREQUENCY IN THE TREATMENT OF FLABBINESS OF THE LABIA PUDENDAS OF MENOPAUSAL WOMEN}

Arthur Hipólito Pereira Leite; Adrielle Nunes de Andrade Silva; Orlando Augusto de Morais Miranda; Maria Aparecida Coelho Lopes

\section{RESUMO}

A flacidez dos grandes lábios vulvares é uma das principais queixas de mulheres, principalmente das que se encontram na pós-menopausa. Entre os vários tratamentos para recuperação do tropismo e do turgor dessa região, a radiofrequência (RF) tem sido eleita por ser um procedimento não invasivo, eficaz no tratamento da flacidez cutânea facial e corporal. Este artigo tem por objetivo avaliar os efeitos da radiofrequência no tratamento da flacidez dos grandes lábios do pudendo da mulher menopausada. As consultas se deram nas bases de dados da PubMed, Scielo, PEDro e Google Acadêmico. Foram selecionadas 83 publicações, entre os anos de 2008 e 2019, das quais 32 foram objetos deste estudo, sendo 21 na língua portuguesa e 11 na língua inglesa. Também fizeram parte do conteúdo do trabalho, informações pesquisadas em 2 livros na língua portuguesa. $O$ tratamento da flacidez dos grandes lábios da vulva com a radiofrequência, tem mostrado bons resultados e boa aceitação pelas mulheres, por ser indolor e oferecer menor tempo de inatividade e o mínimo de efeitos colaterais e/ou adversos.

Palavras-chave: Flacidez da pele. Menopausa. Vulva. Radiofreqüência.

\section{ABSTRACT}

The sagging of the great vulvar lips is one of the main complaints of women, especially those who are postmenopausal. Among the various treatments for the recovery of tropism and turgor in this region, radiofrequency (RF) has been elected as a noninvasive procedure, effective in the treatment of facial and body skin flaccidity. Objective: To evaluate the effects of radiofrequency in the treatment of sagging large lips of the pudend of menopausal women. Method: The queries were made in the indexing databases PubMed, Scielo, PEDro and Google Scholar. A total of 83 publications were selected between 2008 and 2019, of which 32 were the subject of this study, being 21 in Portuguese and 11 in English. Also part of the content of the work, information researched in 2 books in the Portuguese language. Results: Radiofrequency treatment of the large lips of the vulva has shown good results and good acceptance by women, as it offers less downtime and minimal side and / or adverse effects.

Keywords: Flaccidskin. Menopause. Vulva. Radiofrequency. 


\section{INTRODUÇÃO}

A preocupação com a aparência desde a antiguidade, mergulha a vaidade humana em explorações cosméticas e cirúrgicas, na incessante busca da juventude e da perfeição. A razão preponderante dessa busca se deve ao fato de que a pele, nosso órgão mais evidente, sofre um processo dinâmico imutável, causado pela longevidade cronológica. Essa ação costuma ser gradativa irreversível e complexa. Ela ocorre em todos os níveis celulares e atinge todos os sistemas do organismo ${ }^{1,2,3}$.

Dentro do contexto holístico do envelhecimento, o órgão genital também é afetado, sendo a mulher a mais afetada, por causa da menopausa, que mostra, sem pudor, os sinais do tempo em toda sua essência. Nessa fase da vida feminina, ocorrem alterações que vão desde o escurecimento da coloração da vagina até a diminuição da textura e da elasticidade, que resulta em flacidez de pele e músculo, perda de volume da região íntima, atrofia dos lábios vaginais e ressecamento da vagina $^{1,2}$.

Adicionais aos processos fisiológicos temporais que acometem nossa pele, existem, ainda, os danos causados por fatores exógenos vivenciados, como exposição ao sol de forma indevida, processos mecânicos de depilação a cera, gestações, obesidade, tabagismo, alcoolismo, hábitos alimentares inadequados, uso de anticoncepcionais e vestimentas inadequadas, também podem culminar em alterações estéticas nessa região. Alterações genéticas também podem ser motivo de incômodo para as mulheres ${ }^{1,2,3}$.

Como efeito, a questão da aparência e a funcionalidade da genitália feminina acarretam mudanças de comportamento na mulher, que prejudica sua saúde sexual. Isso é preocupante porque afeta diretamente a qualidade de vida da mesma, no que diz respeito ao bem-estar físico, psíquico e social. Para agravar, muitas mulheres ainda vivenciam sofrimentos causados pela insatisfação com a aparência de sua genitália, pela dificuldade ou falta de excitação sexual, dor durante a penetração, incontinência urinária, secura vaginal, desfiguração ou problemas provocados pelo parto, menopausa ou outros fatores 4,5 .

Todavia, com os avanços sociais e quebras de paradigma, o papel das mulheres no comportamento sexual vem sofrendo mudanças culturais significativas, 
especialmente no Brasil, onde o desejo da genitália ideal explica o crescente númerode tratamentos cosméticos e procedimentos vaginais. Como ainda não existe uma definição anatômica padronizada da normalidade para a área genital, procura-se por um modelo estético mais agradável e uma vagina funcional 1,6,7.

Para atingir o objetivo do sentir-se bem com sua genitália, a mulher moderna pode recorrer aos diversos procedimentos estéticos e/ou funcionais, cirúrgicos e não cirúrgicos, conhecidos como rejuvenescimento vaginal ou estética íntima. Os tratamentos não cirúrgicos, têm sido procurados em substituição às cirurgias, por oferecerem menos riscos e incômodos no pós-cirúrgico. Dentre a gama de opções para obtenção de uma genitália satisfatória, a radiofrequência tem se despontado 3,5,8.

O objetivo que norteia essa pesquisa é avaliar se os efeitos da radiofrequência, no tratamento da flacidez dos grandes lábios do pudendo da mulher menopausada, produzem eficácia.

\section{MATERIAIS E MÉTODOS}

A presente pesquisa narrativa foi desenvolvida sob uma abordagem qualitativa, através de um levantamento seletivo e não sistemático da literatura pertinente ao tema, com busca de artigos, revistas especializadas, dissertações e livros, através da mídia impressa e on-line.

Os artigos foram rastreados em banco de dados, utilizando-se os indexadores PubMed, Scielo e Google Acadêmico. Para tanto, os termos de busca em fonte digital, em português e inglês, respectivamente, foram: flacidez da pele, menopausa, vulva, radiofreqüência, flaccidskin, menopause, vulva, radiofrequency.

Para rastrear o máximo de publicações e evitar a repetição dos mesmos, foram realizadas várias combinações de palavras. A princípio, foram consideradas 83 publicações e, destas, 32 foram selecionadas para compor o trabalho, sendo 21 na língua portuguesa e 11 na língua inglesa, publicados entre os anos de 2008 a 2019.

Como critérios de exclusão, foram descartadas 13 publicações por referenciarem tratamentos voltados para a área médica, 16 por utilizarem outros recursos associados à radiofrequência, 19 por apresentarem conflitos de interesse e, 
3 inconclusivas. As bibliografias mais relevantes para inclusão foram determinadas pelos seus conteúdos, na intenção de amparar os aspectos do presente estudo.

\section{RESULTADOS E DISCUSSÃO}

A pele, além de ser o órgão que reveste todo o corpo humano, dá continuidade, revestindo os sistemas: respiratório, digestório e urogenital. Apesar de sua aparência frágil, ela possui funcionalidade tão importante como as de outros órgãos do nosso corpo. Ela é separada em camadas chamadas epiderme, derme e hipoderme e possui como anexos: glândulas sebáceas, sudoríparas, unhas e folículos pilosos ${ }^{5,9}$.

A epiderme, camada mais superficial da pele, é um epitélio pavimentoso e estratificado queratinizado, que apresenta a flacidez cutânea como fator determinante. Ela é formada em sua maior parte por células de queratinócitos, responsáveis por funções importantes, desenvolvidas de acordo com a ação de cada camada, que são cinco: córnea, lúcida, granulosa, espinhosa e basal. Quando as camadas mais superficiais são destruídas, as mais profundas se restauram ${ }^{5,10}$.

A derme se estabelece da epiderme ao tecido subcutâneo e é rica em estruturas fundamentais para funcionalidade da pele. Ela é dividida em duas camadas: papilar e reticular. A papilar é formada por uma fina camada de tecido conjuntivo frouxo, e possui vasos sanguíneos, linfáticos e terminações nervosas. A camada reticular, mais grossa, contém fibras elásticas que possuem papel indispensável na firmeza, elasticidade e sustentação da pele. Essas fibras são formadas por fibroblastos que sintetizam elastina e colágeno, proteína mais abundante no corpo humano e responsável pela integridade e resistência dos tecidos. Sob a derme, encontra-se o tecido adiposo $5,8,10$.

$O$ processo de envelhecimento cutâneo ocorre por fatores intrínsecos e extrínsecos. O fator intrínseco se relaciona com a idade do indivíduo e sua genética e se expressa como uma pele alípica, enrugada, flácida e com apresentação de algumas neoplasias benignas. Histologicamente ocorre redução da espessura da epiderme, atipia nuclear ocasional, diminuição ou aumento do número de melanócitos e das células de Langerhans, que são as células efetoras do sistema imune da pele. 
Já o fator extrínseco está relacionado com a ação dos agentes externos sobre a pele, como exposição solar, agentes químicos e tabagismo 7,8,10,11.

As características da pele foto envelhecida, se devem à substituição das fibras colágenas maduras por colágeno com aparência basofílicas, formando um material constituído de elastina degradada e proteínas microfibrilares ligadas à fibronectina, uma glicoproteína da matriz dérmica. Esse processo declina as funções do tecido conjuntivo e faz com que as camadas de gordura sob a pele não consigam se manter uniforme, podendo acarretar aumento da espessura, rítides proeminentes, alterações discrômicas, telangiectasias, queratoses actínicas, afora a diversidade de lesões elementares benignas, pré-malignas e neoplásicas ${ }^{2,12}$.

As alterações da pele no envelhecimento intrínseco são sutis, com presença de flacidez, rugas finas e palidez cutânea. Isso ocorre porque as fibras de colágeno tipo I, que são encontradas com mais frequência no tecido conjuntivo e tem função de proporcionar a sustentação e integridade da pele, é sintetizado por fibroblastos. Nesse processo, as fibras de elastina, que são proteínas resistentes encontrada na derme e que suportam trações, se ramificam de forma irregular e diminuam sua quantidade, dando lugar ao aumento de células de gordura ${ }^{5,10}$.

A menopausa é um fenômeno biológico antecedida pelo climatério, período em que ocorre a baixa de produção do hormônio estrogênio, decorrente do esgotamento dos folículos ovarianos e diminuição da produção de testosterona pelas glândulas suprarrenais, tornando a menstruação irregular, com menor fluxo e um tempo mais longo entre os períodos menstruais. Ele compreende o período de transição da fase procriativa para a não procriativa, podendo iniciar-se a partir dos 35 anos, variando, para alguns autor 4,9,10.

Para a Organização Mundial de Saúde (1996) a menopausa é a fase da vida da mulher que cessa a capacidade reprodutiva. Os ovários deixam de funcionar e a produção de esteróides e peptídeos hormonais diminuem. Esse evento tem data para começar: a da última menstruação da vida da mulher. Como consequência, o organismo passa apresentar diversas mudanças fisiológicas, sendo algumas resultantes da função ovariana e de fenômenos menopáusicos a ela relacionados e outros devido ao processo de envelhecimento ${ }^{4,9}$. 
A vagina, a vulva, o vestíbulo, os grandes e pequenos lábios e o trígono da bexiga têm uma alta concentração de receptores de estrogênio; portanto, eles são um indicador biológico sensível dos níveis séricos desses hormônios nas mulheres. A perda de estrogênio em mulheres na pós-menopausa produz uma disfunção chamada síndrome geniturinária da menopausa. Com a queda dos níveis dos hormônios sexuais ocorre a diminuição dos nutrientes, das fibras elásticas e de colágeno da mucosa vaginal, alterando a densidade do revestimento da vagina, da uretrae das fibras do tecido conjuntivo que conferem sustentação a essas regiões ${ }^{12,13,14}$.

Essas alterações podem culminar em atrofia vaginal, que atinge cerca de $45 \%$ das mulheres na menopausa. Além do mais, com a redução do volume nos lábios externos o vestíbulo é exposto e propicia a mulher à irritações, coceiras e infecções. Esses sintomas são responsáveis pela queda da qualidade de vida sexual em cerca de 60 a 70\% das mulheres na pós-menopausa e torna a sexualidade delas complexa devido aos fatores físicos, psicológicos, socioeconômicos e culturais 9,12,14,16.

Com o aumento da expectativa de vida mundial, o número de mulheres menopausadas se amplia. Como consequência da evolução etária da população feminina, ocorre o hipoestrogenismo, que afeta a estrutura e a função dos tecidos genitais, por induzir a fusão e hialinização de fibras colágenas e a fragmentação de fibras de elastina. Essa afetação culmina na flacidez cutânea, que causa alterações estéticas dos grandes lábios vulvares e gera desconforto e insatisfação dessa coletividade $^{1,17,18}$.

Após o sexo tornar-se mais importante para as mulheres, com evidência no prazer sexual feminino, foi fomentada uma maior atenção para o corpo. Estudos observam que mulheres com imagem corporal positiva relatam mais atividade sexual, orgasmos, sexo com luzes acesas e maior conforto para se despir na frente do parceiro. Em contra partida, mulheres insatisfeitas com a aparência de sua genitália, referem desconforto, apreensão e constrangimentos que afetam suas relações conjugais e trazem prejuízos para a autoestima e qualidade vida das mesmas 8 ,13.

A genitália externa feminina, denominada vulva, é composto de diferentes estruturas anatômicas, incluindo o mons púbis, grandes e pequenos lábios, clitóris e as aberturas externas da uretra e da vagina. O vestíbulo envolve a abertura da vagina, ou intróito, e a abertura da uretra ou do meato uretral. O períneo é a área que se 
estende debaixo da vulva até o ânus. O clitóris é um órgão erétil que é rico em terminações nervosas. É semelhante ao pênis e sua anatomia só foi realmente compreendida nos últimos 30 anos. O clitóris pode ser dividido em três grandes regiões: glande, corpo e crura ${ }^{3,5,29, \text {. }}$

Os lábios maiores ou grandes lábios do pudendo, são duas pregas cutâneas longitudinais, proeminentes, que se unem anteriormente nas proximidades da sínfise púbica, formando um ângulo agudo denominada comissura anterior e, posteriormente, no centro do períneo, formando a comissura posterior. A fenda ântero-posterior recebe o nome de rima do pudendo. Os grandes lábios são revestidos por epiderme, pêlos, glândulas sudoríparas e sebáceas; contém uma grande quantidade de tecido adiposo e uma delgada camada de músculo liso. Sua face interna tem estrutura histológica semelhante à dos pequenos lábio ${ }^{10,20}$.

Os grandes lábios vulvares são compostos por tecido estratificado queratinizado, apoiado por uma camada de tecido conjuntivo conectivo que vai de frouxo a denso. O tecido frouxo contém fibroblastos, macrófagos, mastócitos, plasmócitos, fibras de colágeno, elastina e reticulares; armazena triglicerídeos; atua na redução da perda de calor por meio da pele e funciona como reserva de energia. O tecido denso contém fibras mais densas e flexíveis, que podem ser modeladas por serem mais fortes; apresenta fibroblastos entre as fibras; fibras de colágeno organizadas de forma irregular e fibras elásticas dando-lhe a capacidade de elasticidade ${ }^{5,13,20}$.

A credita-se que não se deve impor um padrão de beleza para genitália feminina porque há uma grande variação na aparência dos lábios dessa região entre os diferentes grupos de populações existentes. Nas culturas ocidentais, por exemplo, os pequenos lábios simétricos não protuberantes são percebidos como normal. Por conta disso, as mulheres com pequenos lábios salientes frequentemente se sentem dismórficas. Estudos mostram que a aparência da região vulvar considerada normal, aglomera uma grande heterogeneidade de formas, tamanhos e cores, mas não a delimita $^{3,21}$.

Alterações nas características anatômicas da genitália feminina, podem aparecer em qualquer idade, independentemente do nível social. Enquanto cerca de $35 \%$ das jovens sofrem a hipertrofia dos pequenos lábios ou o aumento do monte de 
Vênus, cerca de $65 \%$ a $75 \%$ das mulheres na faixa de idade de 40 e 60 anos podem ter atrofia genital, na sua maioria relacionadas à menopausa. Nesse período, também pode ocorrer flacidez dos grandes lábios, hipercromia da região íntima; ressecamento e alargamento vaginal, sudorese excessiva e incontinência urinária ${ }^{5,10}$.

A flacidez cutânea é uma das alterações estéticas dos grandes lábios vulvares que mais causam desconforto e insatisfação ao público feminino. Registros mostram que a atuação da radiofreqüência nessa área da genitália feminina melhora sua aparência, considerando o número de dobras e distensão que pode haver nessa região. Este efeito é denominado lifting pela radiofreqüência.

A radiofrequência é uma radiação do espectro eletromagnético com frequência entre $30 \mathrm{KHz}$ e $300 \mathrm{MHz}$, utilizada há mais de um século para uma variedade de aplicações médicas, na forma ablativa. Registros mostram que em 1976, com potências mais altas, foi usada para combater células cancerígenas e dores crônicas. Em 1991, seu emprego se deu na realização de corte e cauterização dos tecidos. Hoje, com potências eficientes e frequência entre 0,5 e 1,5 MHz, na forma terapêutica ou não ablativa, é utilizada para fins de rejuvenescimento cutâneo ${ }^{8,11,22}$.

$O$ aparelho de radiofrequência pode ser classificado, de acordo com a quantidade de eletrodos, em monopolar, bipolar ou tripolar. A configuração monopolar consiste no uso de um único eletrodo inserido no circuito, com o paciente funcionando como terra. Na bipolar são utilizados dois eletrodos: um ativo, onde ocorre o aumento de temperatura e consequentemente os efeitos térmicos e o eletrodo passivo, que fecha o circuito da corrente. Na tripolar ou multipolar, baseado em três ou mais eletrodos, um eletrodo atua como pólo2, 22 .

Os efeitos celulares do monopolar e do bipolar são idênticos, diferindo apenas na profundidade de penetração da corrente e nos efeitos colaterais. Na configuração monopolar, a profundidade de ação é maior e os efeitos colaterais são maiores quando comparada ao dispositivo bipolar. O objetivo em todos eles é o mesmo: lesão dérmica com posterior neocolanogênese. Dentre as possíveis reações adversas causadas pelo dispositivo monopolar, pode-se citar: edema e dor na área tratada superiores a um período de 24 horas $^{2,5,22}$.

Ao atravessar o tecido humano, com comprimento de onda em nanômetro, a radiofrequência transforma energia elétrica em calor de três maneiras: por vibração 
iônica, forma mais eficiente de transformar energia elétrica em calor através da friç̧ão e colisão entre os tecidos adjacentes, aumentando a temperatura; por rotação das moléculas dipolares, onde as moléculas de água presentes em nosso corpo provocam atração de cargas opostas e causam colisão entre os tecidos adjacentes e por distorção molecular, onde ocorre mínima conversão de calor pela existência de moléculas e átomos com cargas elétricas neutras ${ }^{13,21,22}$.

Os efeitos fisiológicos causados pela radiofreqüência são o aumento da temperatura, que provoca uma reação instantânea de rompimento de ligações de hidrogênio, alterando a estrutura molecular da tripla hélice do colágeno, desnaturando-o e criando fibras mais grossas e mais curtas, com alteração em diâmetro, espessura e periodicidade, que promovem a retração deste. Após um período de 14 a 21 dias, ocorre uma retração mais gradual, onde o colágeno diminui sua extensibilidade e ativa os fibroblastos com neocolanogênese. Processos inflamatórios, que culminam em cicatrização secundária, reorganizam as fibras colágenas e remodela o tecido cutâneo, diminuindo a flacidez da mesma ${ }^{21,22}$.

Com o calor endógeno, induzido pela radiofreqüência, ocorre também a vasodilatação, que aumenta a circulação arterial, através do aquecimento dérmico profundo e volume distribuído. Esse processo melhora a oxigenação, a nutrição e a acidez dos tecidos; aumenta a drenagem venosa; aumenta a reabsorção de catabólitos; diminui edemas nas áreas com processos inflamatórios; aumenta a permeabilidade da membrana celular, permitindo uma melhor transferência de metabólitos; estimula o sistema imunológico e diminui os radicais livres ${ }^{12}$.

$\mathrm{O}$ colágeno se liquefaz a temperaturas acima de $50^{\circ} \mathrm{C}$. Porém, estudos observaram que, ao manter a temperatura terapêutica do tecido alvo entre $40^{\circ}$ a $45^{\circ} \mathrm{C}$, ocorre formação de novas fibras de colágeno. A temperatura de $38^{\circ}$ a $40^{\circ} \mathrm{C}$ tem sido a mais utilizada para flacidez cutânea, por ser mais tolerada, não devendo ultrapassar $41^{\circ} \mathrm{C}$. Vale frisar que na derme ela estará 2 graus mais elevada que na epiderme. Baixas temperaturas e uma menor quantidade de aplicações podem não ser suficientes para modificações fisiológica ${ }^{2,3}$.

A elevação da temperatura também leva ao aumento de atividade metabólica e enzimática das células. Isso inclui síntese e liberação de mediadores químicos. Ao usar uma temperatura acima de $45^{\circ} \mathrm{C}$, pode ocorrer processo inverso, com a 
vasoconstricção, que diminui a circulação como resposta de defesa. A hiperemia surge como consequência do efeito térmico, em que a vasodilatação promove um aumento da circulação periférica local. Isso só ocorre com o uso de intensidade alta, por um tempo maior de aplicação. Quanto maior a frequência menor o comprimento de onda e maior a capacidade de gerar bioefeitos em condições naturais ${ }^{2,8}$.

Pesquisas com avaliação clínica, histopatológica, microscopia eletrônica e imuno-histoquímica obtiveram resultados satisfatórios dos efeitos da radiofrequência no processo regenerativo da pele, por remodelar o colágeno existente e induzir neocolagênese, neoelastogênese, vascularização e infiltração de fatores de crescimento que conferem ao tecido cutâneo a redução da flacidez e melhora da aparência, com preservação da epiderme ${ }^{8,11,23}$.

Em patologias crônicas, a radiofrequência é indicada em todos os processos degenerativos que impliquem na diminuição ou retardo do metabolismo, irrigação e nutrição. Nas profissões que se dedicam à estética, ela atua com eficácia em fibroedema geloide, flacidez de mamas, alopécia, olheiras, adiposidades, estrias, rugas, manchas, fibroses e flacidez de pele, que é um dos maiores problemas causados pelo envelhecimento. Estudos relatam que a radiofrequência melhora a aparência genital e a função sexual em mulheres com queixa de frouxidão dos grandes lábios, por ser mais eficaz em tecido úmido 2, 22,24.

Adicionado à sua eficácia na recuperação do tropismo e turgor dos tecidos dos grandes lábios vulvares, a radiofrequência terapêutica ou não ablativa é um modelo de tratamento que tem grande aceitação pelas mulheres, por oferecer menor tempo de inatividade e o mínimo de efeitos colaterais e/ou adversos, já que um pósoperatório exige cuidados criteriosos para evitar complicações, como o risco de infecção, má cicatrização, hipersensibidade ou hiposensibilidade. Participantes de diversos grupos de estudos publicados, relataram satisfação com o resultado desse método nos tratamentos de flacidez cutânea de grandes lábios vulvares $8,16,25$.

Para tratar flacidez dos grandes lábios da vulva, o aparelho de radiofrequência é utilizado na configuração bipolar. O eletrodo dispersivo, que tem o potencial de zerovolt, deve ser acoplado no dorso da paciente, geralmente na região lombar, para funcionar como terra. Após, o eletrodo ativo é acomodado na cutis dos grandes lábios, após unção dessa região com hidrogel. Os grandes lábios devem ser afastados dos 
pequenos lábios com uma espátula descartável de madeira, que funcionará como protetor deste segundo. A intensidade deve ser aumentada gradativamente para uma temperatura entre $39-41^{\circ} \mathrm{C}$, conforme limiar da paciente ${ }^{3,10,17}$.

A literatura ainda apresenta divergências quanto à dosimetria, frequência $e$ número de sessões para que a radiofrequência estimule a produção de colágeno e melhore a flacidez cutânea. Estudos que utilizam a forma não ablativa para frouxidão genital, mostram protocolos diferentes, que variam de acordo com o equipamento, número de sessões e o tempo de aplicação. Contudo, a manutenção da temperatura com um termômetro infravermelho, em torno dos $40^{\circ} \mathrm{C}$, durante toda a sessão, possibilita a angiogênese, reconhecida como um evento para fornecer nutrientes necessários para a melhoria da neocolagênese, espessamento da derme e realce do metabolismo dos fibroblastos. O controle da temperatura também evita que a derme seja superaquecida ${ }^{3,10}$.

A energia direcionada pela radiofrequência, não causa dor na em região vaginal, sendo desnecessário o uso de anestésicos, o que torna essa terapia tolerável em temperaturas mais elevadas de forma controlada, reafirmando a segurança e eficácia da mesma. Não foram verificados efeitos adversos sérios em estudos que buscaram avaliar a segurança da radiofrequência não ablativa em região de grandes lábios. Em geral, a incidência de efeitos colaterais é baixa e incluem eritema leve e edema transitórios pós-tratamento, com duração inferior a $24 \mathrm{~h}^{10,8,24}$.

É necessário que haja repetidas aplicações da radiofrequência, para que haja durabilidade dos seus efeitos. O paciente deve ser orientado a tolerar temperaturas quentes, porém, deve relatar ao profissional a sensação de qualquer desconforto. A radiofrequência pode ser aplicada em todos fototipos cutâneos, por sua energia ser independente da interação de cromóforo especifico e não destruir a melanina existente na epiderme $e^{2,10,21,22 .}$

É contraindicado o uso da radiofrequência em indivíduos com transtorno de sensibilidade, peeling químicos, neoplasias, metais intra-orgânicos, osteossínteses, implantes elétricos, marca-passo; sobre glândulas que provoquem aumento de hormônio, grávidas, sobre focos infecciosos, pacientes que estejam ingerindo vasodilatadores ou anticoagulante, hemofílicos e em indivíduos com processos febris. É recomendado não aplicar simultaneamente com outros aparelhos de eletroterapia e 
também retirar correntes, aparelhos eletrônicos e elementos metálicos de perto do aparelho, ${ }^{2,5,10}$.

\section{CONCLUSÃO}

O envelhecimento causa flacidez na pele de homens e mulheres. Essa lassidão, que atinge também os órgãos sexuais, prejudica mais as mulheres, principalmente as menopausadas. Devido à queda de estrogênio, a vagina, a vulva, o vestíbulo, os grandes e pequenos lábios e o trígono da bexiga, que têm uma alta concentração de receptores desse hormônio, passam por alterações indesejáveis. Dentre essas variantes, a flacidez dos grandes lábios da vulva causa alterações estéticas e funcionais que afetam as mulheres, tanto no físico como no psicológico ${ }^{3,7,12}$.

A presente pesquisa se pautou na verificação de resultados apresentados na literatura, sobre o uso da radiofrequência, no tratamento da flacidez dos grandes lábios da vulva das mulheres menopausadas. A partir da investigação da bibliografia selecionada, observou-se que esse método pode ser aplicado na região genital feminina de forma segura e indolor, com resultados positivos e eficazes que permitiram o incremento do desempenho sexual e a recuperação da autoestima das mulheres tratadas com esse recurso, gerando satisfação nas mesmas $^{2,8,11,26}$.

Face à conclusão apresentada, propõem-se mais estudos para certificação da resposta fisiológica que o uso da radiofrequência produz no tratamento dos grandes lábios da vulva da mulher, na pós-menopausa, bem como a duração e a dosimetria do tratamento, principalmente na forma não-ablativa.

Conflitos de interesse: não há.

\section{REFERÊNCIAS}

1. Barros AL. Modalidades Terapêuticas nas Disfunções Estéticas. São Paulo: Phorte; 2015. p. $150-5$.

2. Jurado SR, Silva MEP, Moreno JAN, e entre outros. O laser e o tratamento da flacidez e atrofia vulvo-vaginal-uma revisão integrativa da literatura. Rev. Contemporânea de 


\section{Atenção à saúde}

GO [internet]. 2018 [acessado em 2020 Fev 9]; 46(5):283-291. Disponível em: https://www.febras go.org.br/media/k2/attachments/VolZ46Z-Zn5Z-Z2018.pdf.

3. Fernandes KM, Silva WF, Silva M. Efeito da radiofrequência no tratamento da flacidez genital íntima feminino: revisão bibliográfica. Rev. Saúde em foco [internet]. 2019 [acesso em 2020 Fev 9]; (11):1260-1274. Disponível em: http://portal.unisepe. com.br/unifia/wp-content/uploads/sites/10001/2019/11/EFEITODARADIOFREQU\% C3\%8ANCIA-NO-TRATAMENTO-DA-FLACIDEZ-GENITAL-INTIMA-FEMININO.pdf.

4. Ferreira VN, Carvalho $C$, Silva R, e entre outros. Menopausa: marco biopsicossocial do envelhecimento feminino. Rev. Psicologia \& Sociedade [internet]. 2013 [acesso em 2020Fev8];25(2):410-419.Disponível em: http://www.scielo.br/scielo.php?pid=S0 102$71822013000200018 \&$ script=sci_artext\&t Ing=pt.

5. Nogueira MS. Aplicação da radiofrequência em órgão genital feminino: um procedimento não invasivo no tratamento da flacidez de pele vulvar. Rev. UNIMELauro de Freitas [internet]. 2017 [acessado em Fev 8]; p. 32. Disponível em: https://repositorio.pgsskroton.com.br/handle /123456789/15406.

6. Leal MRD. Função sexual após o tratamento com a radiofrequência em região genital feminina: ensaio clínico randomizado [dissertação] [internet]. Salvador: Escola Bahiana de Medicina e Saúde Pública (Mestrado em Tecnologias em Saúde); 2014. 55p. [acesso em $2020 \mathrm{Fev}$ 9]. Disponível em: https://www.Repositório.bahiana. edu.br:8443/jspui/bitstream/bahiana/80/1/Mariana\%20Robatto\%20Dantas\%20Leal.p df.

7. Lakhani S. Vulvar rejuvenation: exploring common presentations and treatment options. Rev. Journal of Aesthetic Nursing [internet]. 2016 [acessado em 2020 Fev 9]; 5(4):176-180. Disponível em: https://www.magonlinelibrary.com/doi/abs/1 0.12968/joan.2016.5.4.176?af=R\&.

8. Lordêlo $\mathrm{P}$, Leal MRD, Brasil CA, e entre outros. Radiofrequency in female external genital cosmetics and sexual function: a randomized clinical trial. Rev. International Urogynecology Journal [internet]. 2016 [acesso em 2020 Fev 8]; 27:1681-1687. Disponível em: https ://link.springer.com/article/10.1007/s00192-016-3020-x.

9. A vulva humana [internet]. News medical life sciences [acesso em $2020 \mathrm{Fev} 9$ ]. Disponível em: https://www.news-medical.net/health/What-is-the-Vulva-(Portuguese) .aspx>. 


\section{Atenção à saúde}

10. Digesu GA, Tailor V, Preti, Mario P, e entre outros. The energy based devices for vaginal "rejuvenation", urinary incontinence, vaginal cosmetic procedures, and other vulvo-vaginal disorders: An international multidisciplinary expert panel opinion. Rev. Neurourology and Urodynamics Journal [internet]. 2019 [acessado em 2020 Fev 8]; p. 1-4. Disponível em: htt ps://onlinelibrary.wiley.com/doi/abs/10.1002/nau.23927.

11. Leal TP, Santos JAB. Contribuição da radiofreqüência na flacidez genital feminina: uma revisão da literatura. Rev. Multidisciplinar e de Psicologia [internet]. 2019 [acessado em 2020 Fev 8]; 13(45):258-269. Disponível em: https://idonline.emnuven s.com.br/id/article/view/1837.

12. Preti M, Vieira B, Pedro D, Giuseppe A, e entre outros. The clinical role of LASER for vulvar and vaginal treatments in gynecology and female urology: An ICS/ISSVD best practice consensus document. Rev. Neurourology and Urodynamics Journal [internet]. 2019 [acessado em 2020 Fev 8]; p.1-15. Disponível em: https://onlinelibrar y.wiley.com/doi/pdf/10.1002/nau. 23931.

13. Alvarez N, Ortiz L, Vicente V, e entre outros. The effects of radiofrequency on skin: experimental study. Rev. Lasers in Sugery and Medicine Journal [internet]. 2008 [acessado em 2020 Fev 9]; 40:76-82. Disponível em: https://onlinelibrary.wiley.com/ doi/abs/10.1002/ls m.20594.

14. Hashim PW, NIA JK, Zade J, e entre outros. Noninvasive Vaginal Rejuvenation. Rev. Cosmetic Dermatology [internet]. 2019 [acessado em 2020 Fev 9]; 102(4):243-246. Disponível em: https://www.mdedge.com/.

15. Frota TC, Padua JB, Pinto APO, e entre outros. Tratamento com laser e radiofrequência da atrofia vulvovaginal: estudo bibliográfico. Rev. Eletrônica Acervo Saúde / Electronic Journal Collection [internet]. 2018 [acessado em 2020 Fev 9]; 17: Disponível em: https://doi.org/10.25248/reas.e80.2019.

16. Marchitelli C. Vulvar cosmetics. Rev. Spinger Link [internet]. 2019 [acessado em 2020 Fev 9]; 81-89. Disponível em: https://link.springer.com/chapter/10.1007/978-3-31961621-6_10.

17. García IC, Aguilera LL, Martínez EA, Piedrabuena V. Effectiveness of nonhormonal products for the treatment of women with vaginal atrophy. Rev. Oficial de la Sociedad Española de Ginecología y Obstetricia [internet]. 2019 [acessado em 2020 Fev 9]; 


\section{Atenção à saúde}

62(3):230-236. Disponível em: https://sego.es/documentos/progresos/v62-2019/n3/0 5-TO_Eficacia-productos -atrofia-vaginal-ING.pdf.

18. Karcher C, Sadick N. Vaginal rejuvenation using energy-based devices. Rev. International Journal of Women's Dermatology [internet]. 2016 [acessado em $2020 \mathrm{Fev}$ 9]; 2(3):85-88. Disponível em: https://www.sciencedirect.com/science/article/pi i/S2352647516300107.

19. Silva MJ, Paiva ACS, Costa IMM. A vagina pós-orgânica: intervenções e saberes sobre o corpo feminino acerca do "embelezamento íntimo". Rev. Horizontes Antropológicos [internet]. 2017 [acessado em 2020 Fev 9]; 47:259-281. Disponível em: http://www.scielo.br/scielo.php?pid=S0104-71832017000100259\&script=sc i_artt ext.

20. Baptista PV, Silva JL, Moutinho JF, e entre outros. Survey on Aesthetic Vulvovaginal Procedures: What do Portuguese Doctors and Medical Students Think?. Rev. Bras. de Ginecologia e Obstetrícia [internet]. 2017 [acessado em 2020 Fev 9]; 39(8):415423. Disponível em: https://www.thieme-connect.com/products/ejournals/html/10.10 55/s-0037-16 03967.

21. What's next for vaginal 'rejuvenation'? [internet]. Dermatology Times 2019 [acessado em 2020 Fev 9]. Disponível em: <https://www.dermatologytimes.com/journal/dermat ology-tim es-october-2018-vol-39-no-10>.

22. Junqueira LCU, Carneiro JA, Paulo A. Histologia Básica: Texto e Atlas. São Paulo: Guanabara Koogan; 2017. p. 333-5.

23. Günes A, Alinsod RM. A mini-review of aesthetic gynecology and leading gynecology associations' approaches to this issue. Rev. Turkish Journal of Obstetrics and Gynecology [internet]. 2018 [acessado em 2020 Fev 9]; 15(2):105-111. Disponível em: https://www.ncbi.n Im.nih.gov/pubmed/29971188.

24. Brasil CA. Fisioterapia no paciente com desordem da diferenciação sexual: funcionalidade e estética. Rev. Pesquisa em Fisioterapia [internet]. 2017 [acessado em 2020 Fev 9]; 7(1):134-137. Disponível em: https://www5.bahiana.edu.br/index.ph p/fisioterapiaa/article/view/1262.

25. Agne JE. Eletrotermofototerapia. São Paulo: Santa Maria; 2019. p. 220-6.

26. Leal TP, Santos JAB. Contribuições da radiofrequência em flacidez genital feminina: uma revisão da literatura. Rev. Multidisciplinar e de Psicologia [internet]. 2019 
[acessado em 2020 Fev 9]; 13(45):258-269. Disponível em: https://idonline.emnuven s.com.br/id/article/view/183 7/2667. 